\title{
Theory of quasiparticle interference on the surface of a strong topological insulator
}

\author{
H.-M. Guo ${ }^{1,2}$ and M. Franz ${ }^{1}$ \\ ${ }^{1}$ Department of Physics and Astronomy, University of British Columbia, Vancouver, BC, Canada V6T $1 Z 1$ and \\ ${ }^{2}$ Department of Physics, Capital Normal University, Beijing 100048, China
}

\begin{abstract}
Electrons on the surface of a strong topological insulator, such as $\mathrm{Bi}_{2} \mathrm{Te}_{3}$ or $\mathrm{Bi}_{1-x} \mathrm{Sb}_{x}$, form a topologically protected helical liquid whose excitation spectrum contains an odd number of massless Dirac fermions. A theoretical survey and classification is given of the universal features, observable by the ordinary and spin-polarized scanning tunneling spectroscopy, in the interference patterns resulting from the quasiparticle scattering by magnetic and non-magnetic impurities in such a helical liquid. Our results confirm the absence of backscattering from non-magnetic impurities observed in recent experiments and predict new interference features, uniquely characteristic of the helical liquid, when the scatterers are magnetic.
\end{abstract}

PACS numbers: 73.43.-f, 72.25.Hg, 73.20.-r, 85.75.-d

A surface of the three dimensional strong topological insulator (STI) [1 3] is a very special place. Topological invariants that characterize the bulk band structure of the underlying time-reversal invariant crystal guarantee the existence of an odd number of gapless surface states with the characteristic Dirac dispersion. The surface electron spins are aligned in the plane of the surface and point in the direction perpendicular to the momentum vector, as indicated in Fig. 11a. Such arrangement of electron spins and momenta has been termed 'helical liquid' and is predicted to exhibit a number of unusual physical properties 4 -9.

One important property of electrons in the helical liquid, noted early on [1, is the absence of backscattering in the presence of non-magnetic impurities. Since electrons with opposite momenta also have opposite spins, backscattering requires a spin-flip process, which cannot occur in the absence of time-reversal symmetry $(\mathcal{T})$ breaking. This fundamental property of the helical liquid has been recently tested in a series of experiments [1013. using the technique of the Fourier-transform scanning tunneling spectroscopy (FT-STS) applied to the previously discovered STIs $\mathrm{Bi}_{1-x} \mathrm{Sb}_{x}$ and $\mathrm{Bi}_{2} \mathrm{Te}_{3}$ [14, 15. The experimental results are consistent with simple heuristic arguments for electron scattering in the helical liquid.

In this Communication we develop a detailed theory of FT-STS in a helical liquid formed on the surface of a STI. We focus on the characteristic universal physics encoded in the low-energy massless Dirac Hamiltonian [1, 9,

$$
H=v \boldsymbol{\sigma} \cdot(\hat{z} \times \mathbf{p})+\sum_{\alpha=0}^{3} \sigma_{\alpha} V_{\alpha}(\mathbf{r})
$$

describing the surface state of all STIs at probe frequencies $\omega$ tuned sufficiently close to the Dirac point. In Eq. (1) $v$ is the Fermi velocity, $\boldsymbol{\sigma}=\left(\sigma_{1}, \sigma_{2}, \sigma_{3}\right)$ is the vector of Pauli spin matrices, $\sigma_{0}=\mathbb{1}, \mathbf{p}=-i\left(\partial_{x}, \partial_{y}\right)$ is the planar momentum operator (we take $\hbar=1$ ) and $V_{\alpha}(\mathbf{r})$ is the impurity potential in channel $\alpha$.

We study the ordinary tunneling and, with an eye on the future experiments, we make predictions for the
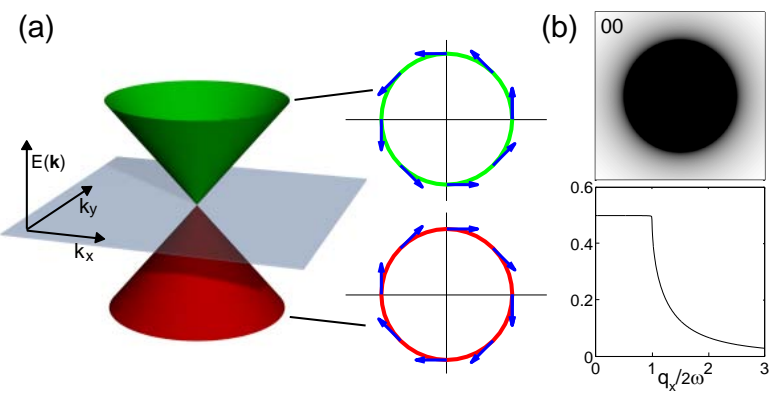

FIG. 1: (Color online) a) Dirac dispersion and spin orientation in helical liquid on the surface of a strong topological insulator. b) FT-STS response $\Im \Lambda_{00}(\mathbf{q}, \omega)$ as a density plot in the $\left(q_{x}, q_{y}\right)$ plane (top), cut along the $q_{x}$ direction (bottom).

spin-resolved FT-STS [16]. We find that non-magnetic impurities produce only weak, non-singular response in the ordinary FT-STS (Fig. 1b), consistent with the expected absence of backscattering. Interestingly, we find that weak magnetic impurities produce no response in the ordinary FT-STS, while strong magnetic impurities produce only weak, non-singular response, except possibly at a resonant frequency. The behavior is even more interesting for a spin-resolved probe. Here, consistent with time reversal symmetry, non-magnetic impurities produce no signal, irrespective of their strength. Magnetic impurities, on the other hand, give rise to distinctive FT-STS patterns with inverse square root singularities at momenta $\left|\mathbf{q}_{\omega}\right|=2 \omega / v$. Depending on the direction of the impurity magnetic moment and the direction in which the probe spin current is polarized we find and classify patterns with pronounced two- and four-fold rotational modulations. Such modulated patterns, in conjunction with future spin-resolved FT-STS experiments, can be used to probe the fundamental properties of the helical liquid as well as the nature of disorder that underlies these patterns. We note that other aspects of magnetic impurities on STI surface have been studied in Ref. 8.

The power of FT-STS technique, originally developed 
TABLE I: Results for FT-STS response function $2 \pi \Lambda_{\alpha \beta}(\mathbf{q}, i \omega)$ evaluated from Eq. (7). Here $\Omega$ represents the ultraviolet cutoff, comparable to the bandwidth of the surface state, $z=2 i \omega /|\mathbf{q}|$ and $\hat{\mathbf{q}}=\left(q_{x}, q_{y}\right) /|\mathbf{q}|$. Functions $\mathcal{F}(z)$ and $\mathcal{G}(z)$ are defined in the text.

\begin{tabular}{|c||c|ccc|}
\hline$\alpha \backslash \beta$ & 0 & 1 & 2 & 3 \\
\hline \hline 0 & $\ln \left(1+\Omega^{2} / \omega^{2}\right)-\mathcal{F}(z)$ & 0 & 0 & 0 \\
\hline 1 & 0 & $\hat{q}_{x}^{2}\left[2+z^{2} \mathcal{G}(z)\right]-1$ & $\hat{q}_{x} \hat{q}_{y}\left[2+z^{2} \mathcal{G}(z)\right]$ & $-\hat{q}_{x} z \mathcal{G}(z)$ \\
2 & 0 & $\hat{q}_{x} \hat{q}_{y}\left[2+z^{2} \mathcal{G}(z)\right]$ & $\hat{q}_{y}^{2}\left[2+z^{2} \mathcal{G}(z)\right]-1$ & $-\hat{q}_{y} z \mathcal{G}(z)$ \\
3 & 0 & $\hat{q}_{x} z \mathcal{G}(z)$ & $\hat{q}_{y} z \mathcal{G}(z)$ & $-\ln \left(1+\Omega^{2} / \omega^{2}\right)-\mathcal{G}(z)$ \\
\hline
\end{tabular}

in the context of simple metals [17] and perfected in the studies of high-temperature cuprate superconductors [18, lies in its ability to use impurities, present in any real material, to probe the electronic response of the underlying ideal clean material at finite momenta q. The experiment measures the local density of states, $n(\mathbf{r}, \omega)$, at a large number $\left(\sim 10^{6}\right)$ of real-space locations $\mathbf{r}$ on the sample surface. The spatial Fourier transform of this signal $n(\mathbf{q}, \omega)$ contains the useful information. Theoretically it can be related to the full electron propagator $G\left(\mathbf{r}, \mathbf{r}^{\prime} ; \omega\right)$ as

$$
n(\mathbf{q}, \omega)=-\frac{1}{\pi} \Im \int d^{2} r e^{-i \mathbf{r} \cdot \mathbf{q}} \operatorname{Tr}[G(\mathbf{r}, \mathbf{r} ; \omega)] .
$$

Here the trace is taken over spin and $\Im$ denotes the strength of a branch cut across the real frequency axis $\Im f(\omega) \equiv[f(\omega+i \delta)-f(\omega-i \delta)] / 2 i$, with $\delta$ a positive infinitesimal. In momentum space the electron propagator has a simple representation in terms of the $\hat{T}$ matrix,

$$
G\left(\mathbf{k}, \mathbf{k}^{\prime} ; \omega\right)=\delta_{\mathbf{k k}^{\prime}} G^{0}(\mathbf{k}, \omega)+G^{0}(\mathbf{k}, \omega) \hat{T}_{\mathbf{k k}^{\prime}}(\omega) G^{0}\left(\mathbf{k}^{\prime}, \omega\right)
$$

where $G^{0}(\mathbf{k}, \omega)=\left[\omega-v\left(k_{x} \sigma_{y}-k_{y} \sigma_{x}\right)\right]^{-1}$ is the unperturbed propagator and the $\hat{T}$ matrix is subject to the Lippman-Schwinger equation

$$
\hat{T}_{\mathbf{k k}^{\prime}}(\omega)=\hat{V}_{\mathbf{k}-\mathbf{k}^{\prime}}+\sum_{\mathbf{q}} \hat{V}_{\mathbf{k}-\mathbf{q}} G^{0}(\mathbf{q}, \omega) \hat{T}_{\mathbf{q k}^{\prime}}(\omega) .
$$

Matrix $\hat{V}_{\mathbf{k}}=\sum_{\beta} V_{\mathbf{k}}^{\beta} \sigma_{\beta}$ is the Fourier transform of the impurity term in Hamiltonian (1).

Below, in addition to the ordinary FT-STS we shall consider also the spin-resolved FT-STS which is obtained by replacing $\operatorname{Tr}\left[\sigma_{i} G(\mathbf{r}, \mathbf{r} ; \omega)\right]$ in the integrand of Eq. (2). Here and hereafter Greek indices run from 0 to 3 while Roman indices run from 1 to 3 . It is useful to consider the quantity $n_{\alpha \beta}(\mathbf{q}, \omega)$ which describes the FT-STS response of a tunneling probe in charge/spin channel $\alpha$ to weak impurities in charge/spin channel $\beta$.

When the impurity potential is weak it is permissible to employ the Born approximation, $\hat{T}_{\mathbf{k k}^{\prime}}(\omega) \approx \hat{V}_{\mathbf{k}-\mathbf{k}^{\prime}}$. In this limit, as first noted by Capriotti et al. [19], the interesting q-dependent part of the FT-STS signal can be expressed in a simple factorized form,

$$
\delta n_{\alpha \beta}(\mathbf{q}, \omega)=-\frac{1}{\pi}\left|V_{\mathbf{q}}^{\beta}\right| \Im\left[\Lambda_{\alpha \beta}(\mathbf{q}, \omega)\right]
$$

where

$$
\Lambda_{\alpha \beta}(\mathbf{q}, \omega)=\sum_{\mathbf{k}} \operatorname{Tr}\left[\sigma_{\alpha} G^{0}(\mathbf{k}, \omega) \sigma_{\beta} G^{0}(\mathbf{k}-\mathbf{q}, \omega)\right] .
$$

Since $V_{\mathbf{q}}^{\beta}$ is a Fourier transform of a random potential one expects it to be a featureless function of $\mathbf{q} . \Lambda_{\alpha \beta}(\mathbf{q}, \omega)$, on the other hand, represents the response of the underlying clean system and contains, in general, prominent features as a function of $\mathbf{q}$ that can be used to directly infer its properties. We now evaluate this response function for our model Hamiltonian (1). Working in the continuum limit and passing to the Matsubara frequency we have

$$
\Lambda_{\alpha \beta}(\mathbf{q}, i \omega)=\int \frac{d^{2} k}{\left(2 \pi^{2}\right)} \frac{L_{\alpha \beta}}{\left[\omega^{2}+k^{2}\right]\left[\omega^{2}+(\mathbf{k}-\mathbf{q})^{2}\right]},
$$

where we also set $v=1$. The factor in the numerator contains the trace of the Pauli matrices and can be expressed as $L_{\alpha \beta}=2 \eta_{\beta} \kappa_{i} \kappa_{j}^{\prime} \operatorname{Tr}\left[\sigma_{\alpha} \sigma_{i} \sigma_{\beta} \sigma_{j}\right]$, where $\kappa=\left(k_{x}, k_{y}, i \omega\right)$, $\boldsymbol{\kappa}^{\prime}=\left(k_{x}-q_{x}, k_{y}-q_{y},-i \omega\right)$ and $\eta_{0,3}=1, \eta_{1,2}=-1$.

Integrals of the type indicated in Eq. (7) are evaluated most conveniently using the technique of Feynman parametrization [20] and have been studied in the context high- $T_{c}$ cuprate superconductors [21] and more recently graphene [22. In fact we may read off the results for $\Lambda_{00}$ and $\Lambda_{33}$ directly from these studies and the remaining integrals can be evaluated in a similar fashion. Our results are summarized in Table I which represents the main result of the present study. They are expressed in terms of two functions,

$$
\mathcal{G}(z)=\frac{2}{\sqrt{-z^{2}-1}} \arctan \frac{1}{\sqrt{-z^{2}-1}},
$$

and $\mathcal{F}(z)=\left(-z^{2}-1\right) \mathcal{G}(z)$ of the dimensionless variable $z=2 i \omega /|\mathbf{q}|$. The results listed in the Table I satisfy

$$
\Lambda_{\alpha \beta}(\mathbf{q}, \omega)=\Lambda_{\beta \alpha}(-\mathbf{q}, \omega),
$$

a property that can be easily established from Eq. (6).

The first line in the table $(\alpha=0)$ describes the ordinary spin-unpolarized FT-STS. The response to nonmagnetic impurities $\Lambda_{00}$, shown in Fig. $1 \mathrm{~b}$, is nonvanishing but weak. In the ordinary metal such response would entail a singularity at momenta $\mathbf{q}_{\omega}$ such that the band energy $\epsilon\left(\mathbf{q}_{\omega} / 2\right)=\omega$, the probe bias. This singularity arises from impurity scattering between states at 
momenta $\mathbf{q}_{\omega} / 2$ and $-\mathbf{q}_{\omega} / 2$. In the present case of helical liquid such backscattering processes are prohibited by time-reversal symmetry [1, 10, 13, and consequently $\Im \Lambda_{00}$ shows only a kink. Interestingly, we find that FTSTS response to magnetic impurities, $\Lambda_{0 i}$, vanishes identically in the Born limit. In this case $\mathcal{T}$ does not prohibit backscattering but the response nevertheless vanishes due to the symmetry property of the integral in Eq. (7). Specifically, Eq. (9) implies that $\Lambda_{0 i}(\mathbf{q}, \omega)=\Lambda_{i 0}(-\mathbf{q}, \omega)$ and we shall see below the that latter must vanish due to $\mathcal{T}$-invariance.

Table I also indicates that response of spin-polarized FT-STS to non-magnetic impurities, $\Lambda_{i 0}$, vanishes. This can be understood as follows. With non-magnetic impurities the surface of STI remains $\mathcal{T}$-invariant and therefore cannot produce a response in the spin channel. We expect this conclusion to remain valid even beyond the Born approximation and beyond the simple linear Dirac model adopted in this study.

Spin-polarized FT-STS in the presence of magnetic impurities, $\Lambda_{i j}$, shows the most interesting behavior. In all cases $\Im \Lambda_{i j}(\mathbf{q}, \omega)$ exhibits an inverse square-root singularity, contained in the function $\mathcal{G}(z)$ Eq. 8), at momenta $\left|\mathbf{q}_{\omega}\right|=2 \omega / v$. In addition, all channels except for $\Lambda_{33}$, show interesting angular dependence on $\mathbf{q}$ with two- and four-fold symmetry. For a general direction of impurity magnetic moment $\hat{\mathbf{m}}$ and the STM tip spin-polarization direction $\hat{\mathbf{n}}$, the singular part of the response can be written compactly as

$$
\Lambda_{\hat{\mathbf{m}} \hat{\mathbf{n}}}^{\mathrm{sing}}(\mathbf{q}, i \omega)=\frac{1}{2 \pi}\left(\hat{\mathbf{m}} \cdot \mathbf{Q}^{*}\right)(\hat{\mathbf{n}} \cdot \mathbf{Q}) z^{2} \mathcal{G}(z),
$$

where $\mathbf{Q}=\left(\hat{q}_{x}, \hat{q}_{y},|\mathbf{q}| / 2 i \omega\right)$ and ${ }^{*}$ denotes complex conjugation. These patterns, displayed in Fig. 2, could be used to identify the dominant source of scattering in future spin-resolved FT-STS experiments. Specifically, the distinctive angular dependence can be exploited to deduce the direction of the magnetic moment of impurities deliberately deposited on the surface of STI [23].

Our result of vanishing responses in $\Lambda_{0 j}$ and $\Lambda_{i 0}$ channels raises the question of whether this conclusion survives beyond the Born approximation. Corrections to the Born approximations may become important when the scattering potential is strong. To this end we now consider the full $\hat{T}$ matrix governed by Eq. 4. For an arbitrary impurity potential it is possible to find the solution only numerically. For isolated point-like impurities it is however possible to obtain exact analytical results. In the following we shall focus on this limit.

For a single impurity characterized by a $\delta$-function real space potential at the origin we have $\hat{V}_{\mathbf{k}}=\sum_{\beta} V^{\beta} \sigma_{\beta}$, independent of $\mathbf{k}$. The solution for the $\hat{T}$ matrix is simplest and most instructive when we consider scattering in a single channel, $\hat{V}_{\mathbf{k}}=V^{\beta} \sigma_{\beta}$ (no summation over $\beta$ ). The $\hat{T}$ matrix is then also momentum independent and can be
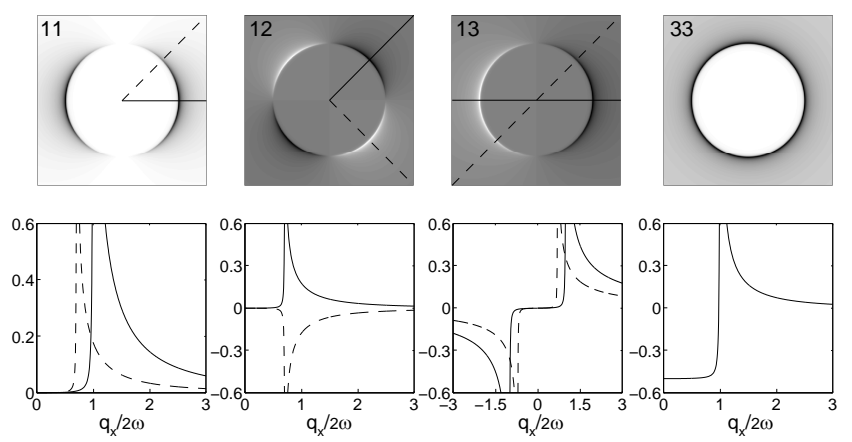

FIG. 2: Spin-resolved FT-STS response $\Im \Lambda_{i j}(\mathbf{q}, \omega)$ to magnetic impurities. Top row gives the density plot in the $\left(q_{x}, q_{y}\right)$ plane in channel $(\mathrm{ij})$ indicated in the upper right corner. Bottom row shows the corresponding cuts along the indicated lines parametrized by $q_{x}$.

written as

$$
\hat{T}_{\mathbf{k k}^{\prime}}^{\beta}(\omega)=\sum_{\alpha} \sigma_{\alpha} T_{\alpha}^{\beta}(\omega)
$$

Substituting this form into Eq. (4) and taking the trace we find an equation for $\hat{T}_{\alpha}^{\beta}(\omega)$ of the form

$$
T_{\alpha}^{\beta}(\omega)=V^{\beta} \delta_{\alpha \beta}+\frac{1}{2} V^{\beta} g_{0}(\omega) \sum_{\mu} \operatorname{Tr}\left[\sigma_{\alpha} \sigma_{\beta} \sigma_{\mu}\right] T_{\mu}^{\beta}(\omega)
$$

where

$$
g_{0}(\omega)=\frac{1}{2} \sum_{\mathbf{k}} \operatorname{Tr}\left[G^{0}(\mathbf{k}, \omega)\right]=-S \frac{\omega}{4 \pi} \ln \left(1-\frac{\Omega^{2}}{\omega^{2}}\right) .
$$

Here $S$ is the area of the STI surface and for finite concentration of impurities it should be replaced by the inverse impurity density $n_{I}^{-1}$. The solution of Eq. 12 reads

$$
\begin{aligned}
& T_{\beta}^{\beta}(\omega)=\frac{V^{\beta}}{1-V^{\beta} g_{0}(\omega)}, \\
& T_{0}^{j}(\omega)=\frac{\left(V^{j}\right)^{2} g_{0}(\omega)}{1-\left[V^{j} g_{0}(\omega)\right]^{2}},
\end{aligned}
$$

and all other components vanish.

Eq. (14) describes the expected resonant enhancement of the scattering potential due to the higher order terms in the Born series. This will affect the amplitude but not the momentum structure of the FT-STS signal.

The result in Eq. 15 is more interesting and informs us that starting at the second order in the Born expansion scattering potential in the $\sigma_{j}$ channel (i.e. magnetic impurities with the moment along direction $j$ ) produces non-zero $\hat{T}$ matrix in the charge channel. Magnetic impurities, therefore, will be visible by the ordinary FT-STS probe, although the signal might generally be weak due to its appearance in the second order of the expansion in the powers of $V^{j}$. Since the $\hat{T}$ matrix does not introduce any new momentum dependence for point-like scatterers 
the momentum-space structure of $\delta n_{0 j}(\mathbf{q}, \omega)$ will be the same as $\delta n_{00}(\mathbf{q}, \omega)$, displayed in Fig. 1 b.

Finally it is worth noting that Eq. 12 implies $T_{i}^{0}(\omega)=$ 0 , confirming our previously stated expectation that nonmagnetic impurities cannot produce a signal in the spinresolved FT-STS. Therefore, $\delta n_{i 0}(\mathbf{q}, \omega)$ remains zero to all orders in the Born expansion, as dictated by $\mathcal{T}$ invariance of the underlying system.

Our results presented above underscore the unique capabilities of the FT-STS technique and its spin-polarized counterpart, applied to the surface of a 3-dimensional strong topological insulator. The expected absence of backscattering in the helical liquid formed on such a surface in the presence of non-magnetic impurities [1, 10 13 is reflected by the non-singular FT-STS response shown in Fig. 17. This stands in a sharp contrasts to the response of the ordinary metal which involves inverse square root singularity when $\epsilon(\mathbf{q} / 2)=\omega$.

In addition to this finding our study reveals several striking and unexpected features when the impurities are magnetic. First, although backscattering is not prohibited in this case, we find that the FT-STS response nevertheless vanishes identically for weak scattering potential treated in the Born approximation. The reason for this unexpected null result is the symmetry property Eq. (9) of the response function $\Lambda_{\alpha \beta}(\mathbf{q}, \omega)$ which equates the relevant response $\Lambda_{0 j}(\mathbf{q}, \omega)$ to $\Lambda_{j 0}(-\mathbf{q}, \omega)$. The latter in turn underlies the spin-polarized response to weak nonmagnetic disorder and must therefore vanish in a $\mathcal{T}$-invariant system. We note that this conclusion does not rely in any way on our assumption of the Dirac Hamiltonian (1) but follows from the general requirement of time reversal invariance and symmetry (9), and will remain valid for arbitrary momenta away from the Dirac point. Indeed this result is applicable to an arbitrary $\mathcal{T}$-invariant system and indicates that the response of ordinary FT-STS to magnetic part of the the scattering potential will generally be weak as it appears only in the second order of the Born expansion.

In order to get strong FT-STS response to magnetic disorder one must employ the spin-resolved tunneling probe [16. In this case the inverse square root divergence at $\epsilon(\mathbf{q} / 2)=\omega$ is recovered. In our Dirac model of STI surface (1) this singular response is accompanied by a distinctive pattern of rotational anisotropy summarized by Eq. 10 which appears when either $\hat{\mathbf{m}}$ or $\hat{\mathbf{n}}$ have a component in the plane of the sample surface.

To model the FT-STS response away from the Dirac point one must include corrections to the Dirac Hamiltonian reflecting the underlying band structure at larger momenta. Very recently, such terms have been considered to model the surface state band structure of $\mathrm{Bi}_{2} \mathrm{Te}_{3}$ and results in good agreement with experiments have been obtained 24, 25]. For other topological insulators such effects will be different as they depend on the details of the underlying crystal structure. Our results, on the other hand, will remain applicable to any STI surface when the probe bias is tuned sufficiently close to the Dirac point and constitute the universal low-energy theory of FT-STS on the topologically protected STI surface states.

Acknowledgment. - The authors benefitted from the discussions with I. Garate and T. Pereg-Barnea. Support for this work came from NSERC, CIfAR and The China Scholarship Council.

[1] L. Fu, C. L. Kane, and E. J. Mele, Phys. Rev. Lett. 98 106803 (2007).

[2] J. E. Moore and L. Balents, Phys. Rev. B 75 121306(R) (2007)

[3] X.-L. Qi, T.L. Hughes, and S.-C. Zhang, Phys. Rev. B 78, 195424 (2008).

[4] L. Fu and C. L. Kane, Phys. Rev. Lett. 100, 096407 (2008).

[5] Y. Ran, A. Vishwanath, and D.-H. Lee, Phys. Rev. Lett. 101, 086801 (2008).

[6] X.-L. Qi and S.-C. Zhang, Phys. Rev. Lett. 101, 086802 (2008).

[7] B. Seradjeh, J.E. Moore, and M. Franz, Phys. Rev. Lett. 103, 066402 (2009).

[8] Q. Liu, C.-X. Liu, C. Xu, X.-L. Qi, and S.-C. Zhang, Phys. Rev. Lett. 102, 156603 (2009).

[9] S. Raghu, S.B. Chung, X.-L. Qi, S.-C. Zhang, arXiv:0909.2477

[10] P. Roushan et al., Nature 460, 1106 (2009).

[11] K.K. Gomes et al., arXiv:0909.0921.

[12] Z. Alpichshev et al., arXiv:0908.0371

[13] T. Zhang et al., arXiv:0908.4136

[14] D. Hsieh et al., Nature 452, 970 (2008).

[15] Y. Xia et al., Nature Phys. 5, 398 (2009).

[16] F. Meier et al., Science 320, 82 (2008).

[17] M.F. Crommie, C.P. Lutz, D.M. Eigler, Nature 363, 524 (1993).

[18] J. Lee, K. Fujita, A.R. Schmidt, C.K. Kim, H. Eisaki, S. Uchida, J.C. Davis, Science 325, 1099 (2009), and references therein.

[19] L. Capriotti, D.J. Scalapino and R.D. Sedgewick, Phys. Rev. B 68, 014508 (2003).

[20] See e.g. M. E. Peskin and D. V. Schroeder, An Introduction to Quantum Field Theory, Addison-Wesley 1995.

[21] T. Pereg-Barnea and M. Franz, Phys. Rev. B 68, 180506(R) (2003); Int. J. Mod. Phys. B 19, 731 (2005).

[22] T. Pereg-Barnea and A.H. MacDonald, Phys. Rev. B 78, 014201 (2008).

[23] H.C. Manoharan, C.P. Lutz, and D.M. Eigler, Nature 403, 512-515 (2000).

[24] X. Zhou, C. Fang, W.-F. Tsai, J. Hu, arXiv:0910.0756.

[25] W.-C. Lee, C. Wu, D.P. Arovas, S.-C. Zhang, arXiv:0910.1668 